\title{
NuklearMedizin 2020: Abstract-Einreichung bis zum 1. November geöffnet!
}

Noch bis zum 1. November 2019 um 14.00 Uhr ist es möglich, Abstracts für die kommende 58. Jahrestagung der Deutschen Gesellschaft für Nuklearmedizin in Leipzig einzureichen.

Der Kongresspräsident, Prof. Dr. Markus Essler (Bonn), und das wissenschaftliche Komi- tee unter der Leitung von Prof. Dr. Michael Schäfers (Münster) freuen sich auf zahlreiche interessante Beiträge.

Sie können Ihre Beiträge online auf der Kongresshomepage nukmed20.nuklearmedizin.de unter „Abstracts“ einreichen. Nach dem Ende der Abstracteinreichung erfolgt die Begutachtung durch das wissenschaftliche Komitee.

Wir wünschen Ihnen viel Erfolg für Ihre eingereichten Beiträge. 